\title{
A Research about Adaptive Subdivision Algorithm Based On Doo- Sabin Mode
}

\author{
Xumin Liu, Yongxiu Xu, Xianpeng Yang and Xiaojun Wang \\ College of Information Engineering \\ Capital Normal University, Beijing 100048, P. R. China \\ 1iuxumin@126.com
}

\begin{abstract}
Subdivision surface method is a series of iterative operation adopts a certain subdivision formula for an initial grid, obtains the smooth limits surface finally, and can dispose any arbitrary complex topology grid. At present most of the subdivision algorithm are 1-4 subdivisions and as the number of subdivision increase, the grid grow so toorapid in the number of patch that it is difficult for the model after subdivision to deal with other things. We proposed an adaptive Doo-Sabin Mode subdivision algorithm to solve this problem, which take the average vector of the vertex and the angle between the intersecting surfaces of the vertex as a measurement criterion. This criterion is used to divide the surface, and then make local subdivision. In this way, when the times of subdivision are fewer (the demand of smoothness is not too high), the effect of subdivision has little difference, but efficiency of the algorithm can be greatly improved. Compared with the normal Doo-Sabin subdivision model, experimental results showed that adaptive Doo-Sabin subdivision algorithm can largely slow the growth speed of the amount of model data on the premise that guarantee the quality of surface.
\end{abstract}

Keywords: surface subdivision, Doo-Sabin Subdivision, quadrilateral mesh, threshold

\section{Introduction}

Through nearly 30 years of research, a large number of surface subdivision algorithms are proposed. In general, the most commonly used subdivision models in subdivision process; make the geometry elements on control meshes exponentially grow, such as Catmull-Clark subdivision [1]. With each subdivision, the number of quadrilateral mesh on the control meshes will become 4 times. Even the $\sqrt{3}$ subdivision mode [2] that grows slower, with each subdivision, the mesh plate number will increase 3 times. When we process the subdivision operation on the model that has large amount of data requires a lot of storage space and computing time to save the geometric elements and topological information after subdivision, which has great impacts on time complexity and space complexity of the algorithm and on the range of algorithms' application. In fact, the geometric model needed to be subdivided is not completely rough, so we usually don't need to subdivide the whole model each time for obtain the smooth limit surface. If we make several subdivision on the relatively uneven area in the model will make these areas become smooth. However, even there have much more subdivisions on the relatively smooth area it cannot obtain more remarkable results. On the contrary, it will increase the amount of unnecessary data, waste resources, reduce the speed of model processing and make the model difficult to be control in the subsequent operations. Adaptive subdivision algorithm [3] can solve those problem, the idea is when the subdivision operation processed on one layer, according to the actual need, used a threshold value criterion to determine which region (relative not flat) continues to participate in next layer subdivision, which region (relative flat) stops subdivision, through this local subdivision both can get ideal smooth limit surface, and can reduce the growth speed of data in 
subdivision process. Adaptive Subdivision can use local grids represent the high precision subdivision surfaces, which make important significance on the practical application.

\section{Doo-Sabin Subdivision Theoretical Basis}

\subsection{Concept and Frame}

Usually, we set up $^{v}$ lowercase letters for control vertices, $e^{e}$ for side of grid, $f$ for mesh surface, $n$ for surface vector. If two surface meshes share an edge, says for edge adjacent surface to each other. The meshes surface set composed of all surfaces that have a public vertex with ${ }^{f}$ surface referred to as the ${ }^{f}$ surface's 1- neighborhood.

Adaptive subdivision framework can be divided into adaptive subdivision criterion, cracks elimination and the calculation of the adaptive mesh vertices three-part. At present, in calculation of the adaptive grids' vertex part, most of adaptive algorithm used the uniform subdivision criteria to calculate. The adaptive algorithm proposed in this paper used Doo-Sabin subdivision algorithm to calculate the position of new vertex.

\subsection{Adaptive Subdivision Geometric Criteria}

Adaptive subdivision used a threshold value criterion to determine which region(relative not flat) continues to participate in next layer subdivision, which region(relative flat) stops subdivision, and then make a local subdivision in the whole of control meshes, trying to use as less as possible mesh surfaces show high precision subdivision surface [4]. Adaptive subdivision criterion also known as threshold criterion, with purpose of determining under which circumstances the mesh surface participate in the lower layer subdivision, so it also is the most important aspect of adaptive subdivision framework. Adaptive subdivision criteria can be divided into geometric criteria and the Non-geometric criteria two kinds. Geometric criterion mainly considers how to minimize the number of subdivision to get limited surface. At present, most adaptive subdivision algorithms adopt geometric criteria. The Non-geometric criteria mainly reflect other requirements except geometric properties, in other words, is a criteria [5] that subdivides in user's interesting areas. Due to the application range of nongeometrical adaptive criteria is narrow, so the most adaptive subdivision algorithm adopts geometric criterion. This paper mainly introduces some geometric criteria.

Though, at present, the method of geometric criteria are many, the main measure methods mainly including angle measure criteria between surface meshes, vertex curvature judge criteria, distance measure criteria between the control meshes vertex and its limit position, distance error measure criteria between mesh surface and the corresponding surface. The following will introduce these measurement criteria, and we will unified use $\varepsilon_{V}$ as a criterion threshold values.

\section{a) Angle Measure Criteria between Surface Meshes}

In adaptive geometric criteria, the angle measure criteria surface meshes criteria is the most widely used criterion. Amresh, Farin, et al., firstly used the dihedral Angle criteria to adaptively subdivide the Loop surface [6]. The basic idea is: set a threshold value, and then judge the Angle between arbitrary triangular surface and the normal of three adjacent triangles on the surface. As shown in Figure 1 (a). Set up $f_{0}, f_{1}, f_{2}$ for three adjacent surface of surface $f$ in the order, $n$ for outer normal vector, $\boldsymbol{n}_{\boldsymbol{i}}$ for outer normal vector of mesh surface $f_{0}, f_{1}, f_{2}$. Define:

$$
\varepsilon_{T}=\max _{i=0,1,2}\left\{\arccos \left(n \cdot n_{i}\right)\right\} \quad i=0,1,2
$$

The $f$ will be referred to as dead-face when $\varepsilon_{T}<\varepsilon_{V}$.Generally dead-face doesn't participate in the lower subdivision, but if its' adjacent surface is not a dead-face, proper 
subdivision maybe also be used to eliminate the cracks. For big templates of subdivision surface, we can improve precision either by increasing adaptive subnet or by reducing $\varepsilon_{V}$.

In addition, literature [7] put the ideas of watershed subdivision technology into algorithm. First, watershed subdivision technology divided surface into several regions as a basis, then users decide which region continue to participate in the lower subdivision. While this approach reduces the difficulty of prophase work, but when the model is volatile, the adaptive subdivision effect of this method is not obvious. Angle measure criteria between surface meshes also applies to other subdivision models, $\mathrm{Xu}$ [8] use this measure criterion proposed adaptive subdivision based on Doo-Sabin subdivision model, but this method use vertex as a benchmark, the rules as shown in Figure 1 (c). After, Liu and others proposed rules that simplified Xu's, also was based on vertex, and mainly calculated the normal vector Angle of two adjacent surfaces. The rules as shown in Figure 1 (d). Domestic, Li Guiqing, Ma Weiyin, et al., [9] proposed that determining a $f$ surface whether can be divided based on the biggest dihedral angle between $f$ surface and all of it's 1- neighborhood surface, as shown in Figure 1 (b).

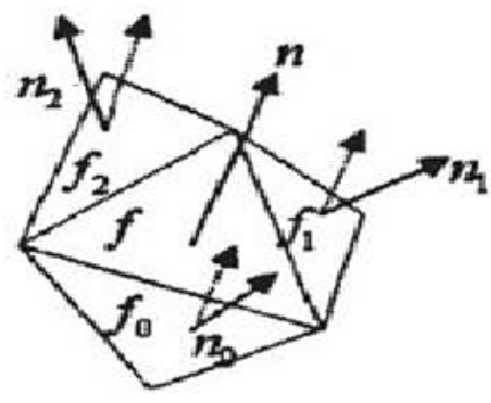

(a) the normal Angle between triangle and it's three adjacent surface

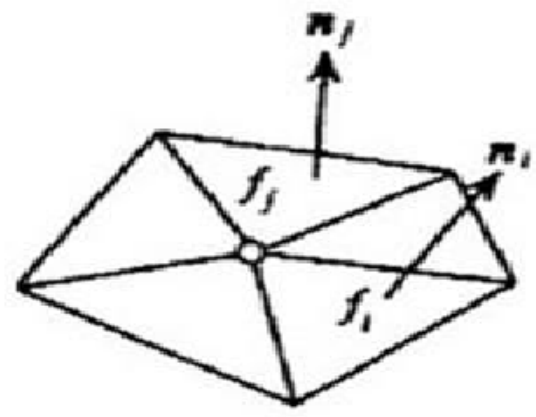

(c) the normal Angle between vertex and all of it's 1-neighborhood

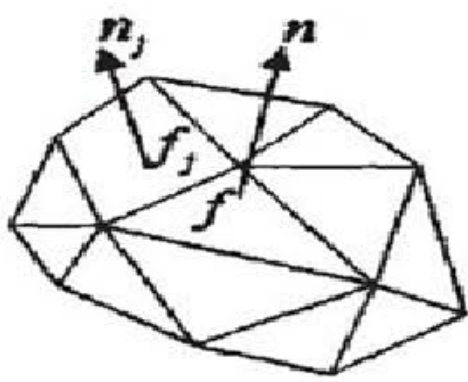

(b) the normal Angle between triangle and it's 1neighbor

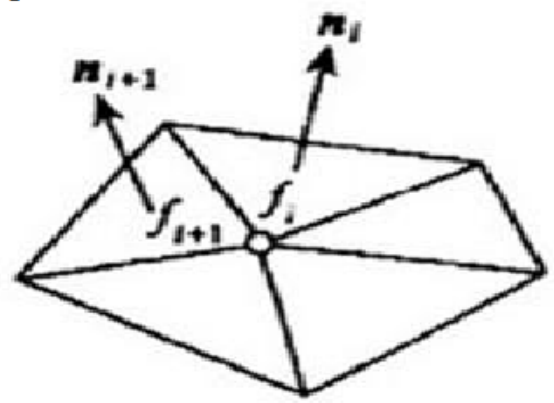

(d) the normal Angle between vertex and all of it's edgeadjacent 1-neighbohood

Figure 1. Based on the Dihedral Angle (or normal) Angle of Adaptive Criteria

\section{b) Vertex Curvature Criteria}

Kobbeft used vertex curvature criterion to implement $\sqrt{3}$ adaptive subdivision mode. Domestic Hu Heping also proposed an adaptive subdivision based on CatmullClark mode, the adaptive process is controlled by the discrete points' curvature $C$. Literature [10] although claimed curvature as measure criterion, in fact, the measure criterion is the angle average value between the vertices and the adjacent side. In actual application, because it is difficult to calculate the curvature of discrete points, and when surface model volatile, the criteria's robustness is poor, therefore, vertex curvature criteria have significant limitations in actual application [11]. 


\section{c) The Mesh Vertices Distance Measurement Criteria}

Mesh vertices distance measurement criteria by controlling the distance between vertex $V$ to its limit position $V^{\infty}$ as a metrics, as shown in Figure 2 (a):

$$
\varepsilon_{V D}=\left|V^{\infty}-V\right|
$$

Muller and others used this criterion to respectively investigate adaptive subdivision of Doo-Sabin mode and Catmull-Clark mode; we can determine a vertex whether continue to participate in the lower segmentation by calculating the distance between the current layer control vertexes to its limit position, each layer segmentation. Obviously, this criterion can't apply to the interpolation subdivision mode. For the interpolation subdivision mode, we can use edge distance error criterion to deal with. Set up $\left(v_{1}, v_{2}\right)$ for a side of surface mesh. It's corresponding vertex $E$ limit for $v_{E}^{\infty}$, so, as shown in Figure 2 (b), define the error criterion is:

$$
\begin{aligned}
\varepsilon_{E}= & \left\|v_{E}^{\infty}-v_{1}^{\infty}-\left|\left(v_{E}^{\infty}-v_{1}^{\infty}\right) \cdot t_{21}^{\infty}\right| t_{21}^{\infty}\right\|, \\
& \boldsymbol{t}_{21}^{\infty}=\left(v_{2}^{\infty}-v_{1}^{\infty}\right) /\left|v_{2}^{\infty}-v_{1}^{\infty}\right|
\end{aligned}
$$

If all sides of the surface $f$ meet $\varepsilon_{E}<\varepsilon_{V}$, it will be no longer subdivision. Mesh vertices distance measurement criterion can more intuitively reflect the subdivision surface approximation degree relative to the dihedral Angle measurement criterion. But this method is not applicable to the interpolation subdivision, the convergence speed is slow and low efficiency. So it is not use as widely as dihedral Angle measurement standards.

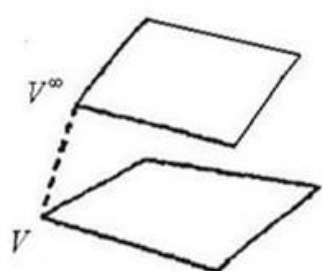

(a)Vertex and its limit distance criterion

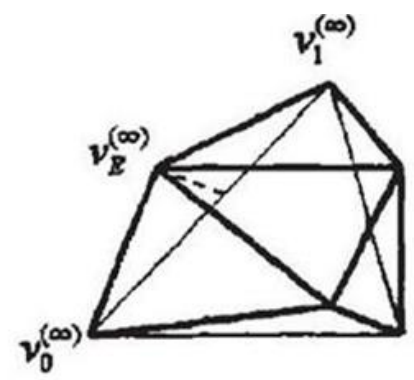

(b)Edge point limit distance criterion

\section{Figure 2. Based on the Distance of the Adaptive Criteria}

\section{d) Distance Error Measure Criteria of between Meshes and the Corresponding Surface}

Dihedral Angle measure criteria and vertex distance criteria are uncontrolled metrics, which they cannot guarantee the approximation error between adaptive mesh and limit surface is less than the given threshold values, so it is difficult to meet the requirements of generating high quality limit surface. In order to solve this problem, some scholars put forward that measuring by computing the error distance between mesh surface and its corresponding mesh surface [12]. But using this measure criterion is on the premise that segmentation mode has corresponding parameter. Set up $f=\left(v_{0}, v_{1}, v_{2}\right)$ for given control triangular mesh, $\boldsymbol{F}=(s, t)$ for linear function of interpolation $f, S(s, t)$ for Loop 
surface of $f$. Introduction: $\varepsilon_{D}(F, S)=\max \{|F(s, t)-S(s, t)|\}$ as approximation error metrics, and apply it to adaptive subdivision for Loop subdivision surface. If $\varepsilon_{D}(F, S)>\varepsilon_{V}$, the triangle mesh is considered to be separable, otherwise, the triangle mesh is inseparable.

Because segmentation mode must have corresponding parameters, which is the precondition of using this criterion, but not all subdivision modes has corresponding parameter, even there are parameters, but error estimate is also very difficult. So in general, this kind of measurement criteria is more complexity and lower efficiency than (1), (2) two kinds of measurement criteria.

Due to the modeling surfaces used for different purposes in practical application, so the subdivision requirements are different. Such as, some require a higher quality of the limit surface, some need to rapid prototyping. For different requirements, we can adopt different measure criteria. For example, if subdivision only requires that the algorithm has high efficiency, and surface quality and accuracy is not high required, Angle measure criteria between surface meshes can be used, if demand high quality surface, we can use the distance measure criteria between the meshes and it's corresponding surface.

\subsection{Cracks Elimination Method}

The generation of cracks is inevitable problems in adaptive subdivided operation, in the process of adaptive subdivision, if two originally adjacent surface, one of them doesn't continue to participate in the lower subdivision, while another surface continue to participate in the lower subdivision, after subdivide operation there will generate cracks in the two adjacent surface, as shown in Figure 3, the shaded part are cracks two adjacent mesh surface generate due to different subdivision depth. Cracks not only let a person produce the visual leak about limit surface, but also affect the smoothness of the limit surface, so we have to eliminate cracks.

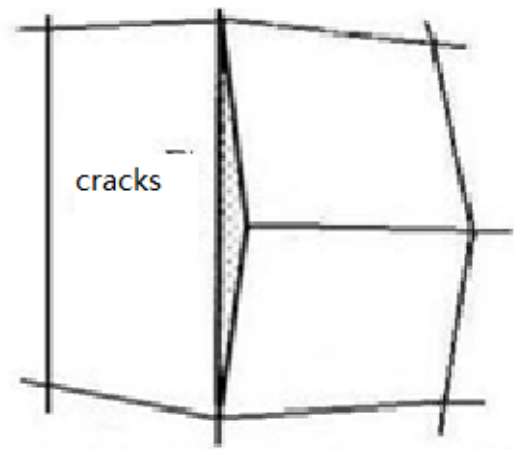

Figure 3. Subdivision Level Inconsistent Caused Cracks

The current crack elimination strategies may be classified according to the following standards:

1) Keep the original mesh type and mixed type: Keep the original mesh type is that the new mesh after the adaptive subdivision generated still maintained the characteristics of original mesh, If the original mesh is a triangle mesh, the new mesh adaptive subdivision generated still is a triangle mesh, as well, if the original mesh is quadrilateral mesh, the new grid generated still is quadrilateral mesh. Mixed type will get a variety of types of polygon after adaptive subdivision, mainly are hybrid meshes composed of triangle meshes and quadrilateral meshes. Although the former strategy has more advantages in keeping features of original meshes, but from the perspective of drawing, there are not much different [13]. 
2) Uniform adaptive subdivision and Non-uniform adaptive subdivision: In adaptive subdivision process, when the two adjacent mesh surface subdivision depth is greater than 1. One method is continuing to do subdivide operation on mesh surface that has smaller depth, until the depth of two mesh surface differ to 1,avoided cracks, this method named uniform adaptive subdivision. This method can get good quality limit surface, but there are cracks migrate problems and exceed subdivision problems. Another strategy is that divided the mesh surface has smaller depth into multiple meshes to avoid cracks, named Non-uniform adaptive subdivided. Although the limit surface generated is not better than former in quality, but there are no cracks migrate problems and exceed segmentation problems.

In practice, while eliminating cracks, we should consider the advantages and disadvantages of various strategies according to the actual situation, therefore we should choose a appropriate cracks elimination strategy.

\section{Adaptive Doo-Sabin Subdivision Method}

\subsection{Basic Concept}

Doo-Sabin Subdivision's [14] basic thought is derived from the Chaibin's cuttingcorner thought, and it extended the quadratic B-spline cutting-angle method to the surface. It was an approximating splitting-point subdivision and applied to the quadrilateral meshes, the surface we obtain in the extreme circumstances is uniform biquadrate B-spline surface.

The first Doo-Sabin subdivision is only for regular quadrilateral mesh surface, then, Doo and Sabin extended this thought to arbitrary topological meshes, and put forward subdivision rules on arbitrary topological meshes. Assuming the vertices of the grid are $P_{i}(1 \leq i \leq N)$.F is an n-Edge surface on the grid, the location of new vertex $p_{i}^{*}$ which is generated by the corresponding vertex of $P_{i}$ is:

$$
p_{i}^{*}=\sum_{j=1}^{n} \alpha_{i j} p_{j}
$$

Where

$$
\alpha_{i j} \begin{cases}\frac{\frac{(n+5)}{5 n}}{3+2 \cos \left(\frac{\pi \pi \hat{i}-j)}{\pi}\right)} & (i=j) \\ \frac{3 n}{S n} & (i \neq j)\end{cases}
$$

The topology rules of Doo-Sabin Subdivision are shown in Figure 4.

New face-face ( $\mathrm{f}-$ face) is the surface constituent by connecting new vertices $p_{i}^{*}$ in order.

New edge-face ( $\mathrm{e}-$ face) is the surface that constituent by the new vertices in the adjacent faces of endpoints of each edge of $p_{i}$.

New vertices-face (v-face) is the surface that constituent by the new vertices in the adjacent faces of vertices of each of $p_{i}$. 


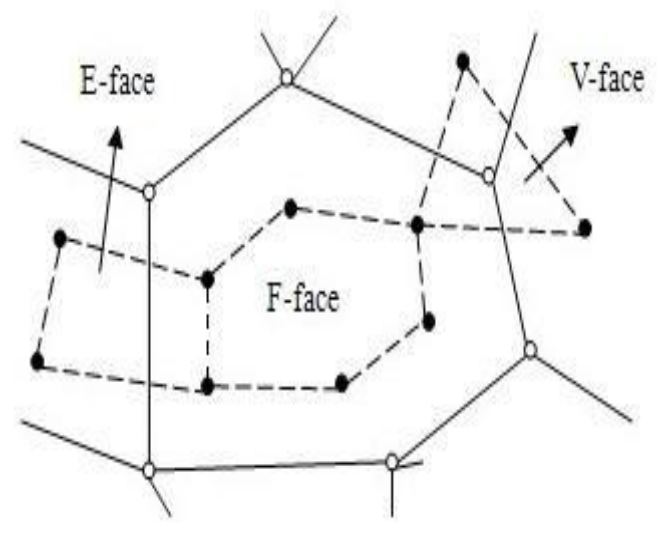

Figure 4. Doo-Sabin Segment Generates Three New Diagrams

Three new surfaces are shown in Figure 4. We can see that after once Doo-Sabin subdivision, the degrees of all vertices of the mesh are 4 , and the number of singular point in the following subdivision process remains unchanged, and it is the sum of the number of non-quadrilateral of initial control grid and the number of vertices' degree less than 4 . The new surfaces are $C^{1}$ continuous except at singular points, and can interpolate the centric of initial control mesh.

To solve the boundary problems of the open-mesh, Doo-Sabin adopts Chaibin methods to calculating. Each vertex is spitted into two new vertices, and finally made boundaries converge to bi-quadratic uniform B-spline.

\subsection{Threshold Calculation}

The thought of adaptive subdivision is that locally subdividing the whole control mesh, and select which area should be subdivided depending on the user's application purpose. In this article, the adaptive Subdivision algorithm selects those relatively uneven areas or high curvature areas to participate in the next layer subdivision. In this paper, based on the Angle geometric criteria between the surface meshes, we proposed a algorithm that dividing surface based on the Angle between average vector of the vertex and intersecting surfaces of the vertex, then make local subdivision. In this way, when the times of subdivision are fewer (the demand of smoothness is not too high), the effect of subdivision has less difference, but efficiency of the algorithm can be greatly improved.

Set up $V^{{ }^{i}}$ for the control vertex of the mesh, and set up ${ }^{n_{i}}$ for the outside normal vector of the control meshes on the 1-neighborhood of $V_{i}, S_{i}$ for area, and the formula of the average normal vector $n_{V}$ of this vertex is:

$$
n_{v}=\frac{\sum_{i=1}^{m} n_{i} S_{i}}{\left|\sum_{i=1}^{m} n_{i} S_{i}\right|}
$$

And, $m$ is the number of polygons in 1-neighborhood of vertex $V_{i}$, as shown in Figure 5 . 


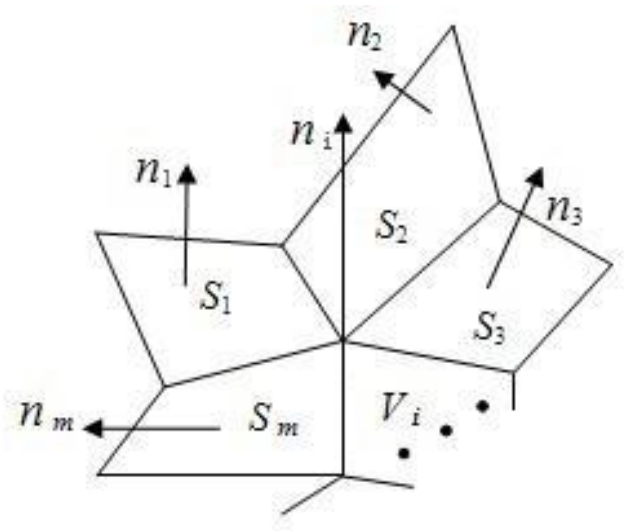

Figure 5. Vertex Average Normal Vector Diagram

Set up $\varepsilon_{T}$ for threshold and $\varepsilon_{V}$ for flatness. The calculation formula is:

$$
\varepsilon_{V}=\max _{i=1,2 \cdots m}\left\{\arccos \left(n_{i} \cdot n_{V}\right)\right\}
$$

The threshold value judging formula is:

$$
\varepsilon_{v}<\varepsilon_{T}
$$

The face $V$ corresponding with the face $V_{i}$ after subdivision is dead-face when the flatness meets the formula (6). If both endpoints of an edge meet the formula (6), the corresponding surface $E$ after subdivision is the dead-face. If the adjacent faces of all edges on the surface $F$ are dead-faces, $F$ will be the dead-face. The dead-face doesn't participate in the next layer subdivision.

\subsection{Algorithm Steps}

The premise of adaptive subdivision is ensuring the surface is smooth. In this paper,

the algorithm controlled adaptive subdivision process by setting the threshold ${ }^{\varepsilon_{T}}$ value. The steps are as follows:

Step1. Traverse the whole surface mesh. Calculate the average vector of all mesh vertices according to the Formula (2).

Step2. Traverse all surface patches. Calculate the flatness $\varepsilon_{v}$ of each vertex according to the Formula (3). Then judge face $\mathrm{V}$, face $\mathrm{E}$ and face $\mathrm{F}$ after subdivision whether are dead-face according to Formula (4). The method to generate the face V, face $\mathrm{E}$ and face $\mathrm{F}$ is as same as Doo-Sabin subdivision.

Step3. The vertices on the alive-face continue to participate in the next layer subdivision, and on dead-face, needn't participate in the lower segment.

Step4. Dealing with cracks generated in the process of subdivision, makes all adjacent grid subdivision depth differ at most once.

Step5. Continue to operate the above subdivision, until it meets the users need.

\subsection{Crack Treatment}

In the process of adaptive subdivision, if a alive - face participate in the lower segment, and if it's adjacent surface is a dead - face, dead-face doesn't participate in the lower segment, along with the subdivision level deepens, there will generate cracks between the alive-face and it's adjacent dead-face. The generation of cracks can destroy the continuity of meshes, so if one surface was subdivided, and its side adjacent surface did not meet the 
criteria for stopping subdivision, cracks will be generated. Cracks can destroy the continuity of meshes, so we must make some corresponding process for the next layer subdivision [15].

In this article, dealing with the cracks generated in the process of adaptive subdivision by using the Non-uniform adaptive subdivision mentioned in 2.3 , means that dividing the surface that has smaller depth in the process of subdivision into multiple surface to eliminate cracks. Specific treatment mainly include Y primitive method, triangle local processing method etc. As shown in Figure 6 (a), the shaded part is cracks, Figure 6 (b) introduce $\mathrm{Y}$ primitive cracks, and Figure $6(\mathrm{c})$ is the introduction of triangle local treatment method to deal with cracks.

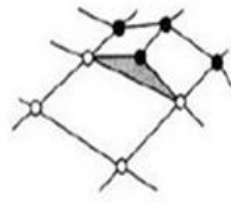

(a)Shadow triangle for cracks

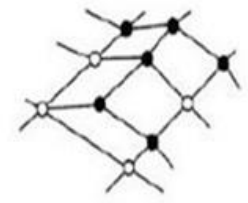

(b) Introduction of primitive $Y$

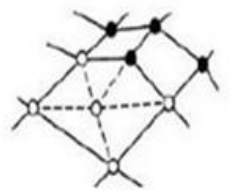

(c) Introduce the triangle subdivision

Figure 6. Cracks Elimination Way

Y primitive method and triangle local processing method can eliminate cracks generated in the process of subdivision, but both of them have advantages and disadvantages. Y primitive method need to consider the neighborhood's subdivision as a whole subdivision, all the inseparable face of neighboring domain was replaced with 1configuration, it's disadvantage is leading to crack migration, but for subdivision depth greater than 1 between adjacent meshes, the triangle local processing method can easily dispose and there will not generate cracks migration, it's disadvantage is that it is easy to produce a exceed segmentation. Through above comprehensive analysis, in this paper, adaptive segmentation algorithm mixed two kinds of crack dispose methods, so we can adopt different processing methods according to different situation. For the mesh surface will be processed, if there is only one side participating in the lower segment, we can adopt triangle local processing method. If there are two adjacent edges participating in the lower segment, we can introduce to $\mathrm{Y}$ primitives, in the case that more than two edges participating in lower segment, we will adopt the normal uniform subdivision algorithm. Therefore we can get optimizing result.

\section{Experimental Evaluation}

Under Windows XP system, we using $\mathrm{C}++$ programming language and OpenGL graphics library in $\mathrm{VC}++6.0$ compiler environment to achieve the algorithm proposed in this paper, and analyzing 3D models. 


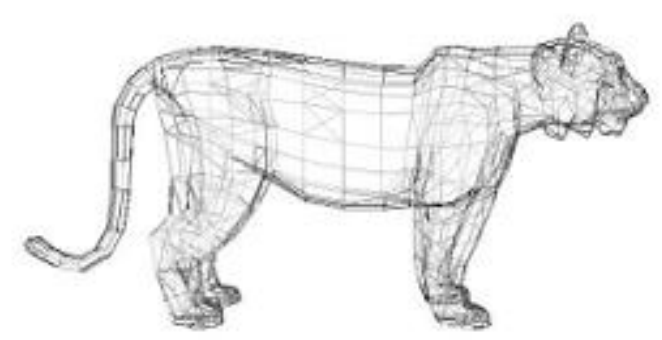

( a) Initial control mesh

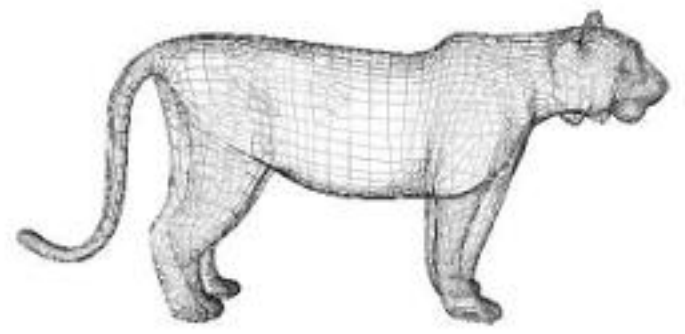

(c) An adaptive subdivision

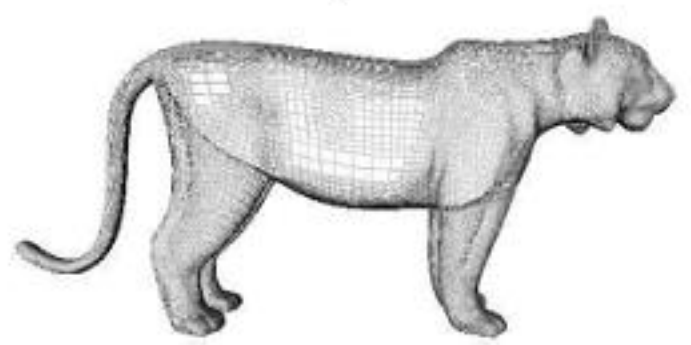

(e) Adaptive subdivision three times

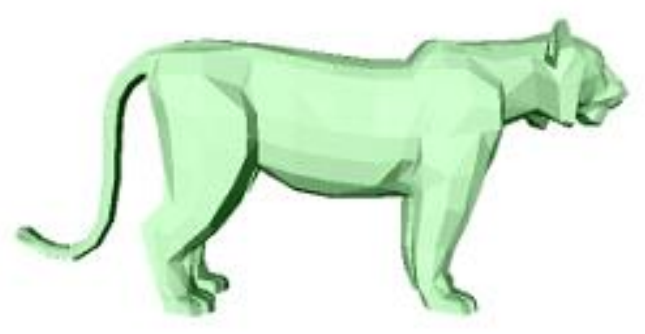

(b) Initial mesh renderings

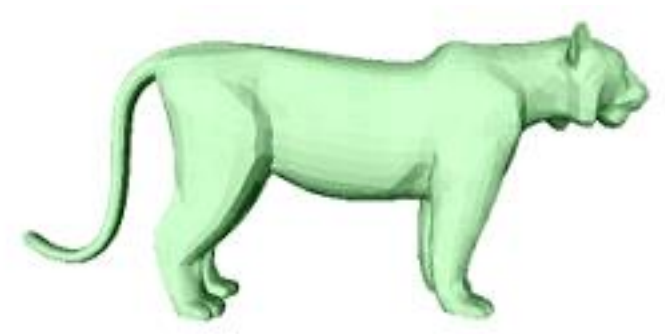

(d) Adaptive subdivision once rende rings

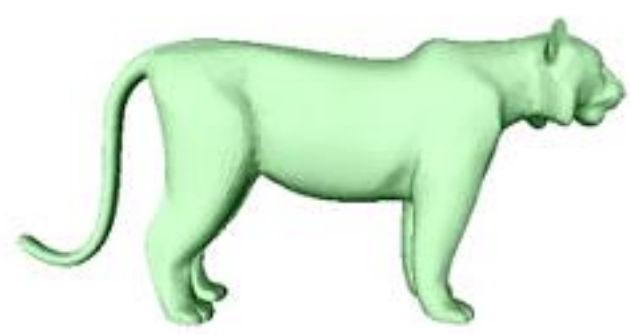

(f) Adaptive subdivision three times rende rings

Figure 7. Leopard Model Adaptive Subdivision Map

Figure 7 shows experimental results of the Leopard model using the adaptive subdivision algorithm in this article. Figure 7 (c), 7(e) respectively shows the result of the Leopard Model was subdivided 1 times and 3 times. We can see that the adaptive subdivision algorithm subdivides the relatively uneven areas and the high curvature areas from the experimental result, so the threshold criteria given in the algorithm can accurately describe some basic features of the leopard model. Such as the front face, ears, neck, buttocks, tail and legs of the leopard model, which are not smooth and have high curvature, are further subdivided, and these areas need to be subdivided more times. In contrast, the relatively smooth areas of the original model are not subdivided further, such as the stomach and other parts. From the table 1 we can see that the number of the initial meshes of the leopard model is 2268. After subdivided by this adaptive subdivision algorithm, the amount of data reduced significantly compared with the normal 1-4 DooSabin subdivision. With the increase of the number of subdivision, the reduction of the number of model data will be more obvious. This makes the processing speed of the adaptive subdivision much faster than the speed of normal subdivision, and the model will be more easily controlled in the subsequent processing. And it can effectively reduce the growth speed of the grids in the subdivision. Therefore, it has great benefit to the application in the graphics transmission and multi-resolution analysis.

Table 1. Leopard Models Adaptive Segmentation of Data and Statistics

\begin{tabular}{c|c|c|c}
\hline Model & $\begin{array}{c}\text { Number of } \\
\text { initial }\end{array}$ & Adaptive Subdivision & $\varepsilon_{\mathrm{T}}$ \\
\hline
\end{tabular}




\begin{tabular}{c|c|cc|c}
\hline & network & Subdivision number & Numbers of segments & \\
\hline $\begin{array}{c}\text { The } \\
\text { Leopard }\end{array}$ & 2268 & 1 & 7216 & 0.05 \\
& & 3 & 39654 & \\
\hline
\end{tabular}

Of course, whichever the adaptive subdivision methods will undermine the overall continuity of subdivision surfaces, and will produce cracks and deterioration. However it's a lack of all adaptive subdivision algorithms and is inevitable. Because in the adaptive segmentation, there must have some surface patches will not participate in the lower subdivision, according to the given criteria, while some surface patches participate in, which making some surface patches at different layers, so it is difficult for us to get high quality adaptive subdivision surface.

\section{Conclusion}

This paper introduces the adaptive subdivision geometric criteria and crack elimination strategy and proposes a adaptive subdivision that judge 2-face threshold base on the Angle between average normal vector of the vertex and this vertex's intersection surface according to feature modeling needs. Using this algorithm not only can represent model with less meshes ,but also can maintain good result characteristics, and it's surface quality is similar to uniform subdivision's. Experimental results show that the algorithm can effectively reduce the storage space of models, slow the growth speed of mesh subdivision process. It has great benefits in graphics transmission, multi-resolution analysis and control applications in the grid.

\section{Acknowledgements}

This research was supported by the National Natural Science Foundation of China General Projects Grant No. 61272029.

\section{References}

[1]. M. Nießner, C. T. Loop and G. Greiner, "Efficient Evaluation of Semi-Smooth Creases in CatmullClark Subdivision Surfaces", Eurographics (Short Papers), (2012), pp. 41-44.

[2]. L. Kobbelt, “ $\sqrt{3}$-subdivision”, Computer Graphics Proceedings, Annual Conference Series, ACM SIGGRAPH, (2000), pp. 103-112.

[3]. Y. Jia, X. Wang and X. Ma, "Local subdivision on triangle mesh", Audio, Language and Image Processing (ICALIP), 2012 International Conference on, IEEE, (2012), pp. 356-359.

[4]. N. A. Husain, M. S. M. Rahim and A. Bade, "Iterative Process to Improve Simple Adaptive Subdivision Surfaces Method for Triangular Meshes", Journal of Computer Science, vol. 7, no, 7 (2011), pp. 11281132.

[5]. F. Wan, F. He and C. Feng, "A simplification algorithm based on appearance maintenance", Journal of Multimedia. vol. 6, no. 5, (2010), pp. 629-638.

[6]. Z. Abderrahim and B. M. Salim, "Interactive Multiresolution Visualization of 3D Mesh", International Journal of Computer Applications, vol. 14, no. 67, (2013), pp. 33-39.

[7]. Y. Zhen-yu, X. Shao-wen and W. Wei-yong, "An Adaptive Loop Subdivision Algorithm for Keeping Local Features with Mesh Segmentation”, E-Product E-Service and E-Entertainment (ICEEE), 2010 International Conference on, IEEE (2010), pp. 1-4.

[8]. Z. Xu and K. Kondo, "Local subdivision Process with Doo-Sabin subdivision surfaces", Proceeding of shape Modeling Internationala, Banff, Albert, (2002), pp. 7-12.

[9]. G. Li, W. Ma and H. Bao, "Fitting System Using Loop Subdivision Surfaces with Sharp Features", Journal of Computer Aided Design \& Computer Graphics, vol. 6, no. 17, (2005), pp. 1179-1185.

[10].K. Zeng, R. Pan and J. Sun, "Algorithm and Implementation of Adaptive Loop Subdivision Surfaces for Preserving Sharp Feature”, Journal of Fujian Normal University (Natural Science Edition), vol. 6, no. 27, (2011), pp. 27-32. 
[11].L. D. Angelo and P. F. Stefano, "Experimental Comparison of Methods for Differential Geometric Properties Evaluation in Triangular Meshes", Computer-Aided Design and Applications, vol. 2, no. 8 (2011), pp. 193-210.

[12].G. Zhou and X. M. Zeng, "Error bounds for Loop subdivision surfaces", International Journal of Computer Mathematics, (2013), (ahead-of-print), pp. 1-16.

[13].M. Nießner, C. Loop and M. Meyer, et al., "Feature-adaptive GPU rendering of Catmull-Clark subdivision surfaces", ACM Transactions on Graphics (TOG), vol. 1, no. 31, (2012), pp. 6.

[14].Y. Liang and D. Wang, "New Method to interplolate image using Doo Sanbin sundivision”, Journal of Computer Application, vol. 6, no. 31, (2011), pp. 723-745.

[15].G. Xie, J. Zhang and C. Huang, et al., "A direct traction boundary integral equation method for threedimension crack problems in infinite and finite domains", Computational Mechanics, vol. 1, no. 53, (2014), pp. 575-586. 